\title{
Up-Regulation of Small GTPases, RhoA and RhoC, Is Associated with Tumor Progression in Ovarian Carcinoma
}

\author{
Akiko Horiuchi, Tsutomu Imai, Cuiju Wang, Satoshi Ohira, Yuzhen Feng, \\ Toshio Nikaido, and Ikuo Konishi
}

Departments of Obstetrics and Gynecology (AH, TI, CW, SO, YF, IK) and Organ Regeneration (TN), Shinshu University School of Medicine, Matsumoto, Japan

\begin{abstract}
SUMMARY: To clarify the role of small GTPases Rho in the biologic behavior of ovarian carcinoma, we first examined the mRNA expression of RhoA, RhoB, and RhoC in benign, borderline, and malignant ovarian tumors using RT-PCR and real-time RT-PCR. The expression and localization of RhoA protein were also analyzed by Western blotting and immunohistochemistry. Finally, we examined whether up-regulation of Rho enhances the invasiveness of ovarian cancer cells in vitro. Analysis of mRNA levels of the Rho family genes revealed that levels of both RhoA and RhoC were significantly higher in carcinomas than in benign tumors (RhoA, $p=0.0035$; RhoC, $p=0.0006$ ). According to histologic subtype, both RhoA and RhoC mRNA levels in serous carcinomas were significantly higher than those in other histologic types. With regard to the International Federation of Gynecological and Obstetrics stage classification, both of RhoA and RhoC mRNA levels were significantly higher in tumors of Stages III+IV than in those of Stages I+II (RhoA, $p=0.0200$; RhoC, $p=0.0057$ ). In addition, analysis of matched pairs of primary and disseminated lesions demonstrated that expression of both RhoA and RhoC mRNA was significantly higher in metastatic than in primary tumors. Examination of the protein level showed that expression of RhoA was also increased in advanced ovarian carcinomas, especially those of serous histology. Accordingly, we hypothesized that up-regulation of Rho GTPases plays an important role in the progression of ovarian carcinoma. Matrigel invasion assay using the ovarian cancer cell line, SKOV3, showed that up-regulation and activation after treatment with lysophosphatidic acid was associated with enhanced invasion of the cancer cells. This increase in invasiveness was suppressed by the addition of C3, a specific inhibitor of Rho. These findings suggest that up-regulation of Rho GTPases is important in the tumor progression of ovarian carcinoma and that Rho family proteins could be a molecular target in cancer therapy. (Lab Invest 2003, 83:861-870).
\end{abstract}

E pithelial ovarian carcinoma is the leading cause of death in female genital malignancies, and more than half of the patients are diagnosed at the advanced stage of disease (National Institutes of Health, 1994). The poor prognosis in patients with ovarian cancer is most likely related to the degree of peritoneal dissemination of cancer cells. The process of peritoneal dissemination is reportedly affected by a variety of gene products: tumor suppressor genes such as p53 and nm23 (Mandai et al, 1995); intercellular adhesion molecules such as cadherins, integrins, and CD44 (Bernstein and Liotta, 1994); proteolytic enzymes such as MMP2 and MMP9 (Schmalfeldt et al, 2001); cytokines such as TNF and IL-8 (Baserga, 1994); and angiogenic factors such as vascular endothelial growth factor and thymidine phosphorylase

DOI: 10.1097/01.LAB.0000073128.16098.31

Received February 28, 2003.

This work was supported in part by a Grant-in-Aid for Scientific Research (to IK) from the Ministry of Education, Science and Culture (No. 13470349), Japan.

Address reprint requests to: Dr. Akiko Horiuchi, Department of Obstetrics and Gynecology, Shinshu University School of Medicine, 3-1-1 Asahi, Matsumoto390-8621, Japan.E-mail: aki9hori@hsp.md.shinshu-u.ac.jp
(Horiuchi et al, 2002; Yamamoto et al, 1997). However, little is known about the molecular aspects of the invasion and migration of ovarian cancer cells: detachment from the primary tumor, attachment to the peritoneum, and invasion into the subperitoneal tissue. Recent attention has focused on the intracellular molecules involved in the enhancement of motility of cancer cells.

The Rho family is ras-related, small guanosine triphosphatases (GTPases), consisting of the Rho, Rac, and CDC42 subfamilies. The Rho family has subtypes designated as RhoA, RhoB, RhoC, RhoD, RhoE, and RhoH genes (Jaffe and Hall, 2002). The Rho family proteins have been known to reorganize cytoskeletons and to regulate the cell migration via activation of effector proteins such as ROCK (Hall, 1998). GTP-bound Rho is known to be an active form, whereas the GDP-bound form is inactive. Rhoguanine nucleotide dissociation inhibitor (Rho GDI) is known to block the conversion between the GTP- and GDP-bound forms (van Aelst and D'Souza-Schorey, 1997). It has been reported that introduction of constitutively active RhoA resulted in transformation of NIH3T3 cells (Avraham and Weinberg, 1989) or in increased invasiveness of rat hepatoma cells (Yoshioka et al, 1998). Overexpression of RhoC could be 
a molecular switch for the metastatic phenotype of human melanoma cells (Clark et al, 2000). Expression of Rho family molecules has recently been reported in breast, lung, pancreas, and colon carcinomas and in testicular germ cell tumors (Fritz et al, 1999; Kamai et al, 2001; Kleer et al, 2002; Suwa et al, 1998; van Golen et al, 1999).

Nevertheless, there have been no available data on the expression of Rho family GTPases in human ovarian neoplasms. In this study, therefore, we examined the mRNA expression of RhoA, RhoB, and RhoC genes at the mRNA level in various epithelial ovarian tumors including benign cystadenomas, borderline malignant tumors, and invasive carcinomas. In advanced ovarian carcinomas, the expression of RhoA and RhoC mRNA was compared between the primary and metastatic lesions. Expressions of RhoA, ROCK, and Rho GDI were also examined at the protein level by Western blotting and immunohistochemistry. Based on observations in vivo, we hypothesized that overexpression of RhoA and RhoC is deeply involved in the dissemination metastasis of ovarian cancer cells. To test this hypothesis, we further examined in vitro the change in invasiveness of human ovarian cancer cells after the up-regulation of Rho GTPases using lysophosphatidic acid (LPA) (Schmalfeldt et al; 2001; Skubitz, 2002).

\section{Results}

Expression of RhoA and RhoC mRNA Is Higher in Ovarian Carcinoma, Especially of Serous Histologic Type, than in Benign Cystadenoma

Representative profiles of RT-PCR products for RhoA and RhoC mRNA are shown in Figures 1 and 2, respectively. An intense band for RhoA mRNA was observed at $183 \mathrm{bp}$ after the RT reaction to RNA extracts from ovarian carcinomas, whereas a faint or weak band was observed in benign or borderline malignant tumors (Fig. 1a). The ratios of RhoA glyceraldehyde-3-phosphate dehydrogenase (G3PDH) in benign tumors, borderline malignant tumors, and carcinomas were $0.04 \pm 0.12,0.16 \pm 0.21$, and $0.24 \pm$ 0.24 (mean $\pm \mathrm{SD}$ ), respectively (Fig. 1b). The levels of RhoA mRNA were significantly higher in carcinomas than in benign tumors $(p=0.0035)$. According to the histologic subtypes of ovarian carcinoma, the ratios of RhoA/G3PDH in serous, endometrioid, clear cell, and mucinous carcinomas were $0.38 \pm 0.23,0.10 \pm 0.15$, $0.08 \pm 0.12$, and $0.22 \pm 0.34$, respectively (Fig. 1c). The RhoA mRNA levels in serous carcinomas were significantly higher than those in endometrioid $(p=0.0389)$ or clear cell carcinomas $(p=0.0075)$.

Bands for RhoB mRNA at $189 \mathrm{bp}$ were detectable only in eight cases of carcinoma (data not shown). There was no correlation between the expression of RhoB and the histologic subtype of ovarian carcinoma.

Bands for RhoC mRNA were clearly detected in various ovarian tumors. An intense band was observed at $181 \mathrm{bp}$ after the RT reaction to RNA extracts from ovarian carcinoma, whereas the band was faint in benign tumors (Fig. 2a). The ratios of RhoC/G3PDH in benign tumors, borderline malignant tumors, and carcinomas were $0.02 \pm 0.01,0.15 \pm 0.20$, and $0.27 \pm$ 0.20 , respectively (Fig. 2b). The levels of RhoC mRNA were significantly higher in carcinomas than in benign tumors $(p=0.0006)$. According to the histologic subtypes of carcinoma, the ratios of $\mathrm{RhoC/G3PDH}$ in serous, endometrioid, clear cell, and mucinous carcinomas were $0.49 \pm 0.09,0.22 \pm 0.14,0.14 \pm 0.15$, and $0.15 \pm 0.26$, respectively (Fig. 2c). The RhoC mRNA levels in serous carcinomas were significantly higher than those in other histologic subtypes (endometrioid, $p=0.050$; clear cell, $p=0.0027$; mucinous, $p=0.0350)$. There was a positive correlation between the mRNA levels of RhoA and RhoC (correlation coefficient, $\rho=0.89$ ) and no correlation between RhoA and RhoB nor between RhoB and RhoC.
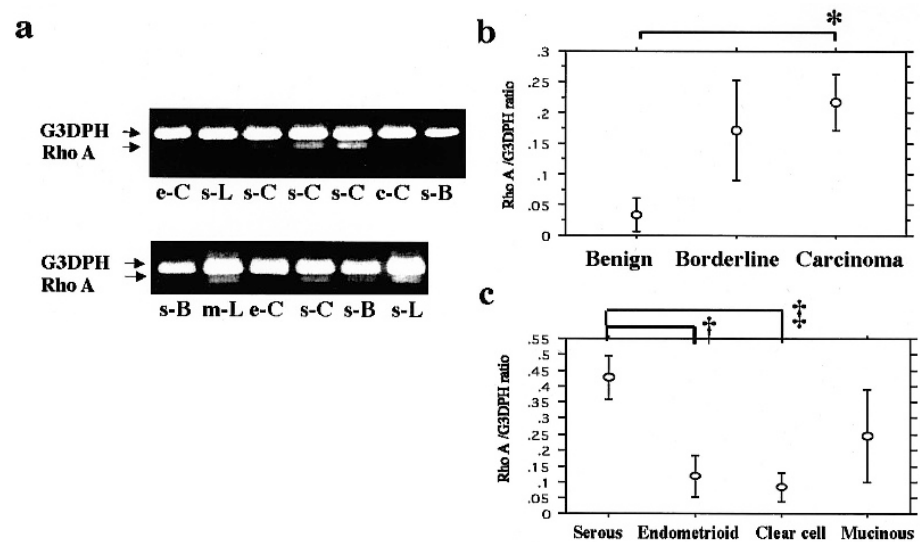

Figure 1.

Expression of RhoA mRNA in epithelial ovarian tumors. a, Expression of RhoA mRNA by RT-PCR. Specific bands for RhoA and glyceraldehyde-3-phosphate dehydrogenase (G3PDH) are observed at the expected 183 and 226 bp, respectively. b. The ratio of RhoA/G3PDH in ovarian carcinomas is significantly higher than that in benign tumors. $c$, According to the histologic subtypes of ovarian carcinoma, expression of RhoA mRNA is higher in serous carcinomas than in endometrioid or clear cell carcinomas. $B=$ benign tumor; $L=$ borderline tumor; $C=$ carcinoma; $S=$ serous; $m=$ mucinous; $e=$ endometrioid; $c=$ clear cell; open circle \pm bar $=$ mean $\pm \mathrm{SE} ;{ }^{*} p=0.0035, \dagger p=0.0389, \ddagger p=0.0075$. 

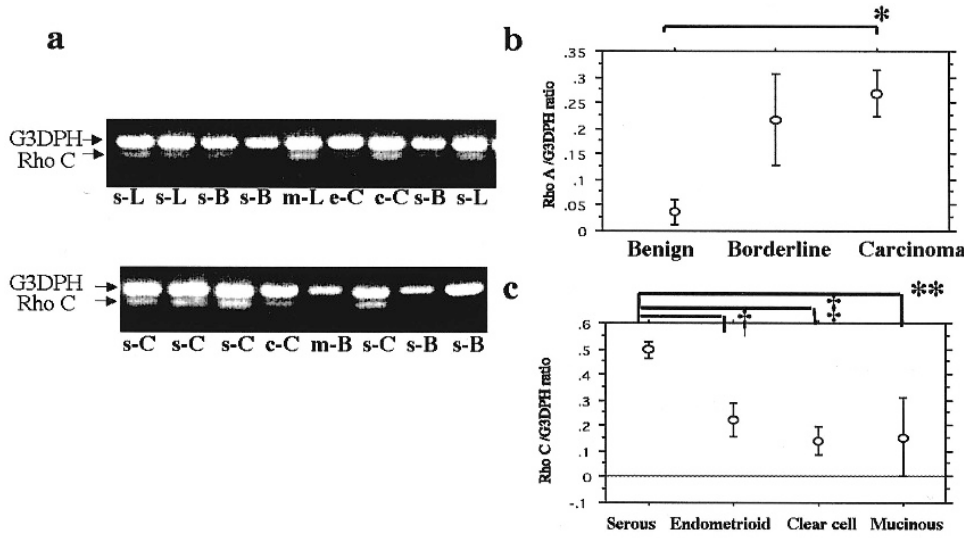

\section{Figure 2.}

Expression of RhoC mRNA in epithelial ovarian tumors. a, Specific bands for RhoC and G3PDH are observed at the expected 181 and 226 bp, respectively. b, The ratio of RhoC/G3PDH in ovarian carcinomas is significantly higher than that in benign tumors. c, According to the histologic subtypes of ovarian carcinoma, expression of RhoC mRNA is higher in serous carcinomas than in endometrioid, clear cell, or mucinous carcinomas. ${ }^{\star} p=0.0006,{ }^{\star \star} p=0.035, \dagger p=0.050, \ddagger p=0.0027$.

To confirm the results of RT-PCR, the mRNA levels of RhoA and RhoC were also analyzed quantitatively using real-time PCR. The expression of both RhoA and RhoC in ovarian carcinomas was significantly higher than that of benign tumors (RhoA, $p=0.0132$; RhoC, $p=0.0467$ ) (Fig. 3).

\section{Expression of RhoA and RhoC mRNA Is Increased in Ovarian Carcinomas of Advanced Stage, Especially in Their Metastatic Lesions}

According to the International Federation of Gynecological and Obstetrics (FIGO) stage classification of ovarian carcinoma, the ratios of RhoA/G3PDH in cases of Stages I+II and III+IV were $0.10 \pm 0.13$ and $0.35 \pm 0.26$, respectively. The ratios of RhoC/G3PDH in tumors of Stages I+II and III+IV were $0.16 \pm 0.16$ and $0.39 \pm 0.22$, respectively. Accordingly, the RhoA and RhoC mRNA levels were significantly higher in tumors of FIGO Stages III+IV than those of Stages I+II (RhoA, $p=0.0200$; RhoC, $p=0.0057$ ) (Fig. 4).

Analysis of matched pairs of primary and metastatic lesions from six cases of ovarian carcinoma showed that expression of both RhoA and RhoC mRNA was significantly higher in metastatic lesions of peritoneal dissemination than in the respective primary tumors (RhoA, $p=0.0260$; RhoC, $p=0.0273$ ) (Fig. 5).
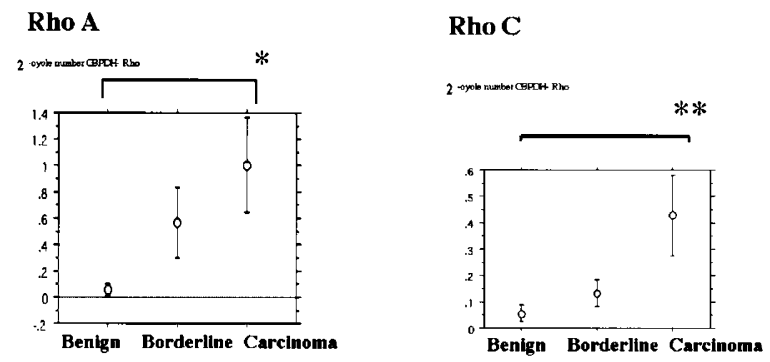

\section{Figure 3.}

The results obtained from real-time PCR, showing that mRNA expressions of both RhoA and RhoC in ovarian carcinomas are significantly higher than those in benign ovarian tumors; ${ }^{*} p=0.0132,{ }^{* \star} p=0.0467$.
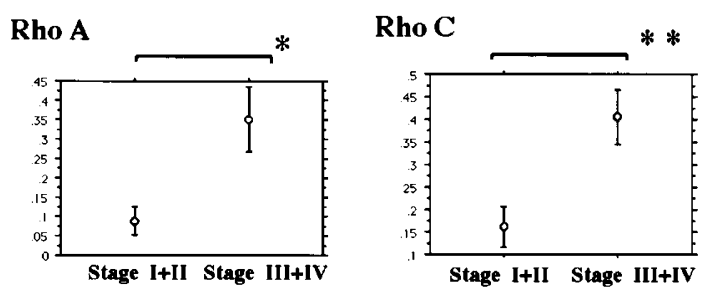

Figure 4.

mRNA expression levels of both RhoA and RhoC in ovarian carcinomas of International Federation of Gynecological and Obstetrics (FIGO) Stage III+IV tumors are significantly higher than those in Stage I + II tumors; ${ }^{*} p=$ $0.0200,{ }^{* \star} p=0.0057$.

\section{Protein Level Expression of RhoA Is Also Increased in Ovarian Carcinoma, But Not of ROCK and Rho GDI}

Analysis of RhoA expression at the protein level by Western blotting revealed a band at $21 \mathrm{kDa}$ (Fig. 6). The bands of RhoA protein in benign and borderline tumors were either below the limit of detection or only faintly detectable. In contrast, most carcinoma cases exhibited a high level of RhoA protein expression. Bands of ROCK at $160 \mathrm{kDa}$ were observed in all cases of ovarian tumors, and there were no apparent differences according to the histologic classifications of benign, borderline, or malignant. Bands of Rho GDI at either 27 or $28 \mathrm{kDa}$ or both were detected in all cases of ovarian tumors. There were no relationships between the levels of Rho GDI expression according to the histologic classification.

Immunohistochemical analysis confirmed the overexpression of RhoA in ovarian carcinomas. Representative profiles of immunostaining for RhoA are shown in Figure 7. Specific staining for RhoA was observed in the cytoplasm of tumor cells. The correlation between RhoA immunostaining and clinicopathologic findings are summarized in Table 1. The immunostaining of RhoA in benign tumors was negative in $60 \%$, weak in $30 \%$, and moderate in $10 \%$ of the cases, and that in borderline malignant tumors was negative in $27 \%$, weak in $55 \%$, and moderate in $18 \%$ of the cases. There were no cases with strong immunostaining in 
Rho A
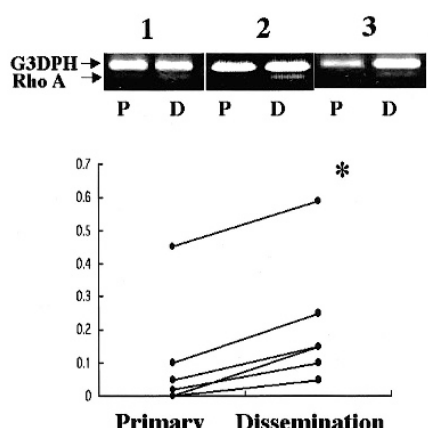

Rho C
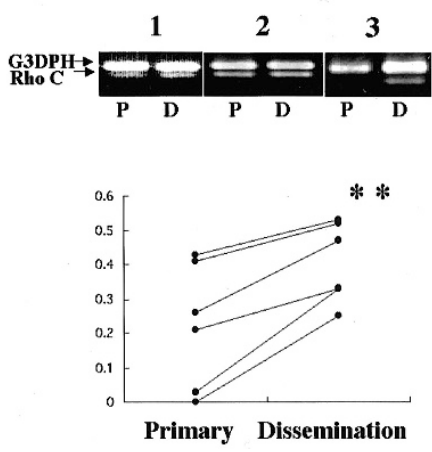

Figure 5 .

mRNA expression levels of both Rho $\mathrm{A}$ and Rho $\mathrm{C}$ in disseminated lesions are significantly higher than those in the respective primary lesions of advanced ovarian carcinoma. $P=$ primary lesion; $D=$ disseminated lesion; ${ }^{*} p=0.0260,{ }^{* *} p=0.0273$.

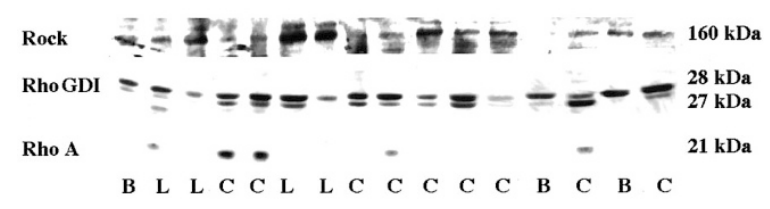

Figure 6 .

Western blotting of RhoA, ROCK, and Rho GDI in benign, borderline, and malignant ovarian tumors. The bands of RhoA protein are observed at $21 \mathrm{kDa}$ in ovarian carcinoma cases but not in benign tumors. In contrast, bands of ROCK at $160 \mathrm{kDa}$ and Rho GDI at either 27 or $28 \mathrm{kDa}$ or both were observed in all cases of ovarian tumor.

benign and borderline tumors. In contrast, RhoA immunostaining in ovarian carcinomas was negative in $31 \%$, weak in $28 \%$, moderate in $25 \%$, and strong in $15 \%$ of the cases (Fig. 7). Accordingly, the staining intensity of RhoA was significantly higher in ovarian carcinomas than that in benign tumors $(p=$ 0.003).

According to the histologic subtypes of ovarian carcinoma, strong immunostaining of RhoA was observed in $8(35 \%)$ of the 25 serous, $1(5 \%)$ of the 18 endometrioid, $0(0 \%)$ of the 16 clear cell, and $1(14 \%)$ of the 7 mucinous carcinomas. The intensity of immunostaining in serous carcinomas was significantly higher than that in other histologic subtypes $(p=$ 0.001).

With regard to the FIGO stage classification, positive immunostaining for RhoA was observed in $23(58 \%)$ of the 40 Stage I+II cases and in $23(85 \%)$ of the 27 Stage III+IV cases. RhoA protein expression was significantly higher in tumors of FIGO Stages III+IV than those of Stages I+II $(p=$ 0.002)(Table 1). In addition, a closer observation revealed that, in the same cases of ovarian carcinoma, the tumor cells invading the stroma showed stronger immunostaining of RhoA than those of lumen formation (Fig. 8, a and b). Histologically, the cancer cells with stronger expression of RhoA tended to be separated from each other and associated with desmoplastic stromal reaction. In 13 of the 21 pairs of primary and metastatic lesions, the tumor cells in the peritoneal dissemination showed stronger immunostaining of RhoA than those in the primary tumor (Fig. 8, c and d); in the remaining 8 cases, the staining intensity did not differ between the 2 lesions.

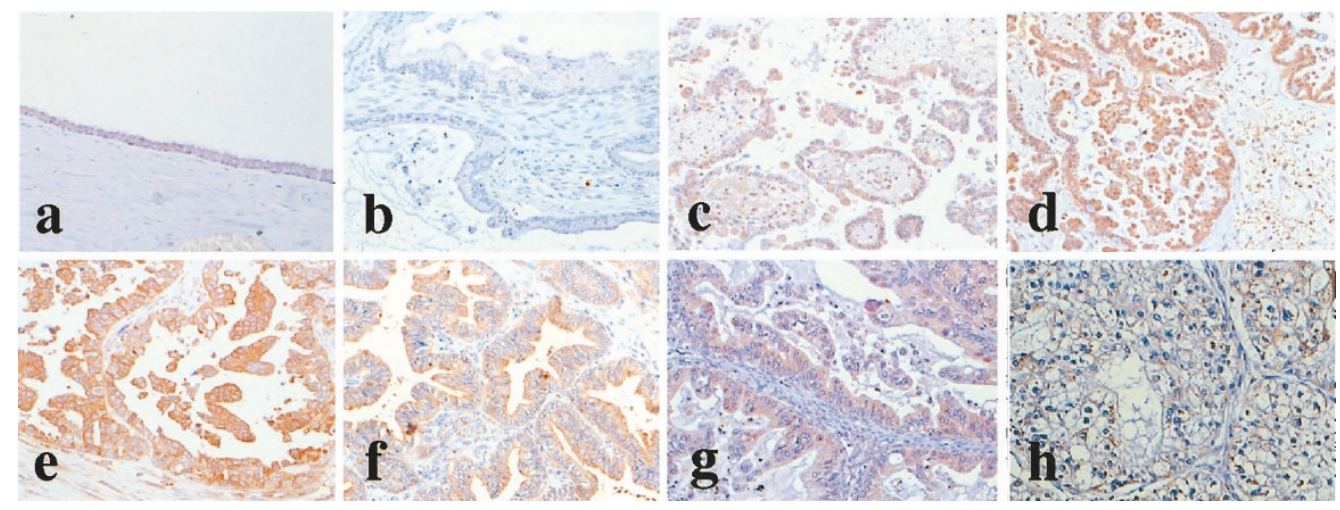

Figure 7.

Immunohistochemical staining of RhoA in epithelial ovarian tumors. The specific staining for RhoA was observed in the cytoplasm of the tumor cells. The immunostaining of RhoA was negative in benign tumor such as serous (a) and mucinous (b) cystadenomas and weak in borderline malignant tumors (c). There were a few cases of serous borderline tumor showing moderate immunostaining for RhoA (d). In contrast, moderate or strong immunostaining for RhoA was frequently observed in ovarian carcinomas of serous (e), mucinous (f), endometrioid (g), and clear cell (h) histology. Original magnification, $\times 200$. 
Table 1. RhoA Immunostaining in Epithelial Ovarian Neoplasms

\begin{tabular}{|c|c|c|c|c|c|}
\hline & \multirow{2}{*}{$\begin{array}{c}\text { Total number } \\
\text { of cases }\end{array}$} & \multicolumn{4}{|c|}{ RhoA immunostaining } \\
\hline & & - & + & ++ & +++ \\
\hline Benign cystadenomas & 20 & $\begin{array}{r}12 \\
(60 \%)\end{array}$ & $\begin{array}{r}6 \\
(30 \%)\end{array}$ & $\begin{array}{r}2 \\
(10 \%)\end{array}$ & $\begin{array}{r}0 \\
(0 \%)\end{array}$ \\
\hline Serous & 6 & 3 & 1 & 2 & 0 \\
\hline Mucinous & 14 & 9 & 5 & 0 & 0 \\
\hline Borderline tumors & 22 & $\begin{array}{r}6 \\
(27 \%)\end{array}$ & $\begin{array}{r}12 \\
(55 \%)\end{array}$ & $\begin{array}{r}4 \\
(18 \%)\end{array}$ & $\begin{array}{r}0 \\
(0 \%)\end{array}$ \\
\hline Serous & 7 & 1 & 4 & 2 & 0 \\
\hline Mucinous & 11 & 4 & 5 & 2 & 0 \\
\hline Carcinomas & 67 & $\begin{array}{r}21 \\
(31 \%)\end{array}$ & $\begin{array}{r}19 \\
(28 \%)\end{array}$ & $\begin{array}{r}17 \\
(25 \%)\end{array}$ & $\begin{array}{r}10 \\
(15 \%)\end{array}$ \\
\hline Histologic type & & & & & \\
\hline Serous & 25 & 4 & 7 & 6 & 8 \\
\hline Mucinous & 7 & 2 & 1 & 3 & 1 \\
\hline Endometrioid & 18 & 7 & 5 & 6 & 1 \\
\hline Clear cell & 16 & 8 & 6 & 2 & 0 \\
\hline FIG0 stage & & & & & \\
\hline 1 & 33 & 13 & 11 & 7 & 2 \\
\hline II & 7 & 4 & 1 & 2 & 0 \\
\hline III & 22 & 3 & 5 & 6 & 8 \\
\hline IV & 5 & 1 & 2 & 2 & 0 \\
\hline
\end{tabular}

The intensity of cytoplasmic staining of RhoA was scored as strong $(+++)$, moderate $(++)$, faint $(+)$, or negative $(-)$, by comparison to the positive internal control.

\section{Up-Regulation and Activation of RhoA and RhoC by LPA Treatment Increases the Invasiveness of Ovarian Cancer Cells In Vitro}

First, we examined the mRNA expression of RhoA and $\mathrm{RhoC}$ in the ovarian cancer cell line, SKOV3, and then we treated the cells with LPA, a bioactive phospholipid known to activate Rho GTPases (Goetzl and An, 1998). LPA has also been reported to be present in ascites and tumor fluids of ovarian cancer (Westermann et al, 1998; Xu et al, 1995b). Real-time PCR showed that LPA treatment upregulated the mRNA expression of RhoA and RhoC (Fig. 9a). In addition, ADP ribosylation assay revealed that LPA treatment increased the amount of GTP-binding form of Rho, ie, activation of Rho proteins occurred (Fig. 9b). Then, we examined whether up-regulation of Rho stimulates the invasiveness of SKOV3 ovarian cancer cells, using a Matrigel invasion assay. The cells were incubated for 20 hours in the absence or presence of $10^{-7}$ or $10^{-6} \mathrm{M}$ LPA in the lower compartment of the culture chambers. The count of cells that passed through the Matrigel-coated membranes showed that the invasive capacity of the tumor cells was significantly stimulated by LPA treatment in a dose-dependent manner (Fig. 9, $c$ and d). Finally, to confirm the involvement of the Rho pathway in the stimulation of invasion by LPA treatment, we used C3, a specific inhibitor of Rho. When the SKOV3 cells were pretreated with $0.1 \mu \mathrm{g} / \mathrm{ml} \mathrm{C} 3$ for 24 hours, the LPA-induced enhancement of cell invasion was completely suppressed (Fig. 9, c and d).

\section{Discussion}

The present study demonstrated that, among various epithelial ovarian neoplasms, the mRNA expression of both RhoA and RhoC obtained by RT-PCR was significantly higher in invasive carcinomas than in benign cystadenomas. The expression levels of RhoA and RhoC in borderline malignant tumors were intermediate between those of benign and malignant tumors, but the differences were not significant. Quantitative analysis of the levels of RhoA and RhoC mRNA using real-time PCR confirmed the higher expression of both RhoA and RhoC in ovarian carcinomas compared with benign tumors. In addition, our analyses using Western blotting and immunohistochemistry showed that the protein level expression of the RhoA was also significantly higher in ovarian carcinomas than in benign tumors. In human neoplasms, there have been reports on the overexpression of the RhoA protein in breast, lung, and colon carcinomas (Fritz et al, 1999), RhoA mRNA in testicular germ cell tumors (Kamai et al, 2001), RhoC mRNA in pancreas carcinomas (Suwa et al, 1998), and RhoC mRNA and protein in breast cancer (Kleer et al, 2002; van Golen et al, 1999). To our knowledge, this is the first report on the expression of Rho GTPases in epithelial ovarian tumors. Our study also demonstrated that there was no difference in the expression of ROCK (the effector protein of Rho) or in the expression of Rho GDI (inhibitor for conversion between active and inactive forms of Rho) among various ovarian tumors. Collectively, our data indicate that both RhoA and RhoC are overexpressed at the 


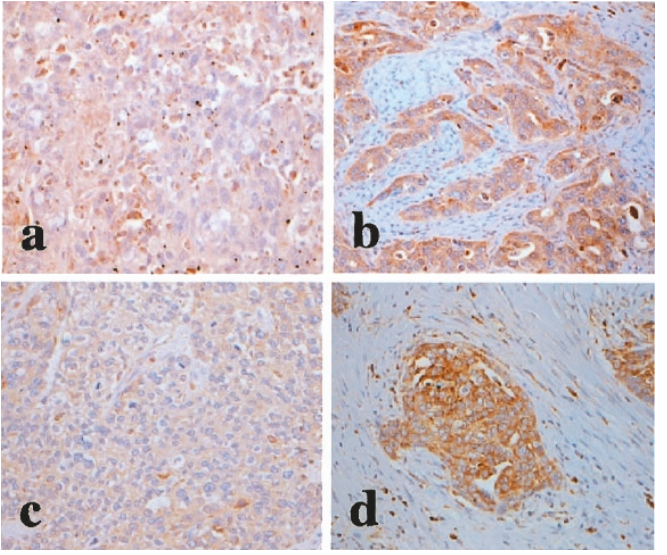

Figure 8.

Immunohistochemical staining of RhoA in carcinoma cells of the primary lesion and in those invading the stroma or those in the disseminated site. In the same case of serous carcinoma ( $a$ and $b$ ), compared with the tumor cells of the primary lesion (a), those invading the stroma (b) showed stronger immunostaining for RhoA. In another same case of serous carcinoma (c and d), compared with the primary lesion (c), the tumor cells in the peritoneal dissemination (d) showed stronger staining for RhoA. Original magnification, $\times 200$.

transcriptional level in ovarian carcinomas, compared with benign tumors.

This study also demonstrated that, among various histologic types of ovarian carcinoma, the expression levels of RhoA and RhoC mRNA as well as RhoA protein were higher in serous carcinoma, which is more frequently associated with peritoneal dissemination, than in other histologic types (Morrow and Curtin, 1998). In addition, according to the FIGO stage classification, the expression levels of RhoA and RhoC
mRNA and RhoA protein were significantly higher in tumors of Stages III and IV than in those of Stages I and II. This seems to be consistent with previous reports showing that the overexpression of Rho was correlated with higher stage in other malignancies (Fritz et al, 1999; Kamai et al, 2001; Suwa et al, 1998). Furthermore, our analysis of Rho expression in matched pairs of primary tumor and its peritoneal dissemination from six cases of advanced ovarian carcinoma showed that both the RhoA and RhoC mRNA levels were significantly higher in the metastatic than in the primary lesions. Immunohistochemical examination also revealed that the staining intensity for RhoA was stronger in the tumor cells invading the stroma or in the peritoneal dissemination than in the tumor cells of the primary lesion. In rat noninvasive hepatoma cells, overexpression of RhoA has been reported to induce invasive behavior (Yoshioka et al, 1999). Overexpression of RhoC has also been reported to promote invasive behavior in melanoma cells (Clark et al, 2000) and in mammary epithelial cells (van Golen et al, 2000). Accordingly, we hypothesized that the overexpression of RhoA and RhoC might be essential in tumor progression, especially in the peritoneal dissemination of ovarian carcinoma.

To test the above hypothesis, we performed an in vitro experiment using the serous ovarian carcinoma cell line, SKOV3. To up-regulate the expression of Rho, we used LPA, which has been known to activate Rho by interacting with specific cell surface G-protein-coupled receptors of the endothelial differentiation gene (Edg) subfamily (Goetzl and An, 1998). LPA is also known to be present in tumor fluid, ascitic fluid
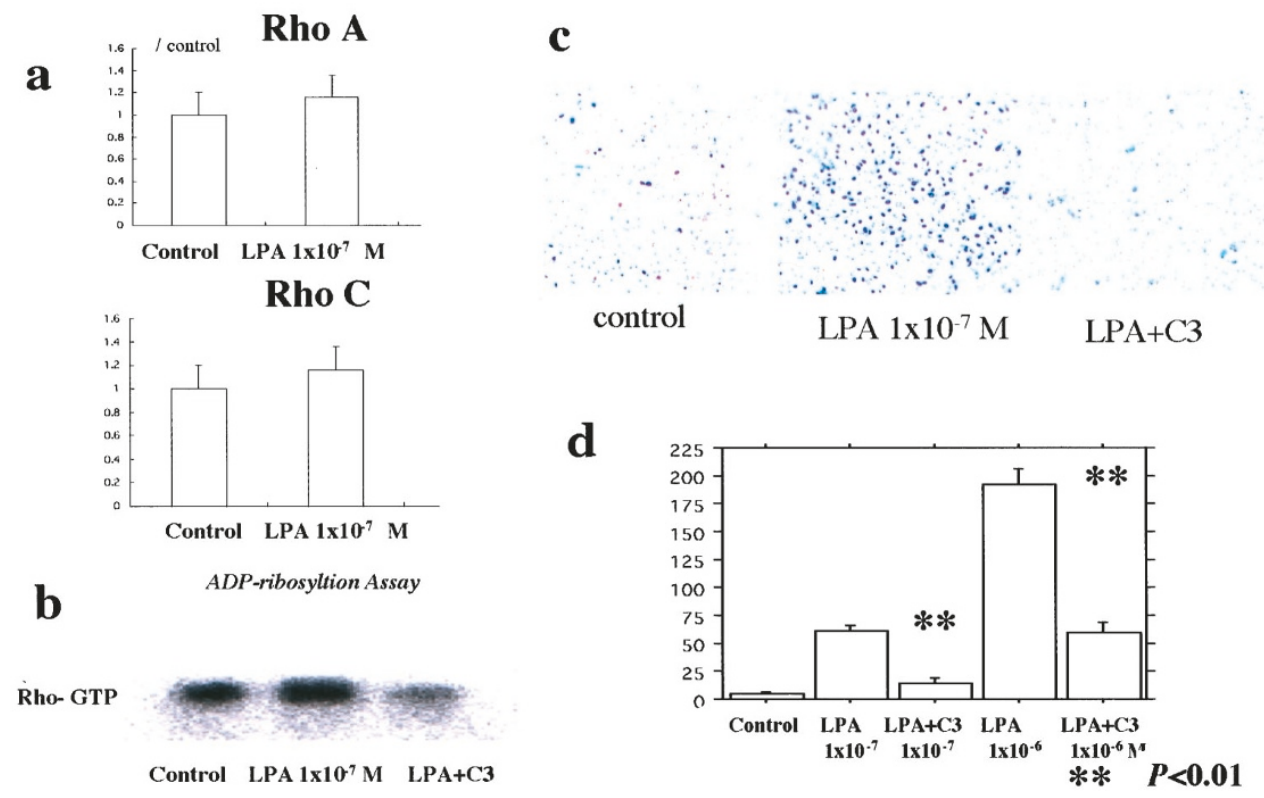

Figure 9.

In vitro experiment using the SKOV3 ovarian cancer cell line, showing that up-regulation and activation of Rho GTPases increases its invasiveness. a, In real-time PCR, expressions of both RhoA and RhoC mRNA are up-regulated by lysophosphatidic acid (LPA) treatment. b, ADP ribosylation assay shows that LPA treatment increases the amount of GTP-binding form of Rho, and this activation is suppressed by addition of C3, a specific inhibitor of Rho. C, Photographs showing the cells that have passed through the Matrigel-coated membranes under LPA treatment with or without C3. Original magnification, $\times 100$. $d$, The number of cells invading the Matrigel is increased by LPA treatment in a dose-dependent fashion, and it is inhibited by C3 treatment. 
(Xu et al, 1995b), and plasma (Xu et al, 1998) of ovarian cancer patients; it works by increasing proliferation (Xu et al, 1995a), invasion (Fishman et al, 2001), and vascular endothelial growth factor production (Hu et al, 2001). Our experiment showed that LPA treatment increased the expression levels of RhoA and RhoC as well as the GTP-binding form of Rho GTPases. Invasion assay demonstrated that up-regulation and/or activation of Rho GTPases by LPA treatment resulted in enhanced invasion of SKOV3 cells through the Matrigel. The enhancement of invasiveness under LPA treatment might not be ascribed solely to the activation of Rho GTPases. The biologic effects of LPA should be carefully evaluated not only with relevance to Rho GTPases but also with other signaltransduction pathways. In our study, however, the enhancement of invasion was suppressed by C3 exotransferase, a specific inhibitor of Rho GTPases.

These in vivo and in vitro findings suggest that up-regulation of Rho GTPases plays an important role in tumor progression, including peritoneal dissemination of ovarian carcinoma. In addition, we recently found that, in SKOV3 ovarian cancer cells, hepatocyte growth factor also activates the Rho GTPase, along with an enhancement of in vitro invasiveness, which is reversed by treatment with C3 (our unpublished observation). Rho GTPase could be an important molecular target in the treatment of ovarian carcinoma in the near future.

\section{Materials and Methods}

\section{Tissue Collection}

Fresh surgical specimens were obtained from 42 women with epithelial ovarian tumors who underwent surgery at the Department of Gynecology, Shinshu University Hospital. Specimens were stored at $-80^{\circ} \mathrm{C}$ for RT-PCR and Western blotting. Adjacent tissues were fixed in $10 \%$ phosphate-buffered formalin, embedded in paraffin, and prepared for hematoxylin and eosin staining. Specimens stained with hematoxylin and eosin were reviewed by I. K. and A. H. to confirm the histopathologic diagnosis using standard criteria (Scully et al, 1998). Histopathologically, 12 were benign cystadenomas, 7 were borderline tumors, and 23 were carcinomas, including 7 serous, 3 mucinous, 5 endometrioid, and 8 clear cell carcinomas. According to FIGO classification, 10 cases were classified as Stage I, 3 were Stage II, 8 were Stage III, and 2 were Stage IV. Both primary and metastatic lesions were available in six cases.

For immunostaining of RhoA, tissues of 109 cases of epithelial ovarian tumors were collected from the pathology files of the Department of Gynecology, Shinshu University Hospital. None had received preoperative chemotherapy or radiotherapy. Histopathologically, 20 were benign cystadenomas, 22 were borderline tumors, and 67 were carcinomas. Of the 67 carcinomas, 25 were serous, 7 were mucinous, 18 were endometrioid, and 16 were clear cell carcinomas. According to FIGO classification, 33 cases were clas- sified as Stage I, 7 were Stage II, 22 were Stage III, and 5 were Stage IV. Of the advanced carcinoma cases, immunoreactivity for RhoA was compared between primary and metastatic lesions in 21 cases. Each tissue was used with the approval of the Ethical Committee of Shinshu University, after obtaining written consent from the patients.

\section{Total RNA Extraction}

Total RNA was extracted by the acid guanidiniumphenol-chloroform method as described previously (Chomczynski and Sacchi, 1987; Horiuchi et al, 1998). One microgram of total RNA was treated with $1 \mathrm{U} / 10$ $\mu$ l of DNase I (Life Technologies, Gaithersburg, Maryland).

\section{RT}

RT was performed using an RNA PCR Kit (Takara Shuzo, Otsu, Japan), with $1 \mu \mathrm{g}$ of RNA sample being added to $20 \mu$ l of a reaction mixture consisting of 10 $\mathrm{mm}$ Tris- $\mathrm{HCl}$ (pH 8.3), $50 \mathrm{~mm} \mathrm{KCl,} 5 \mathrm{~mm} \mathrm{MgCl}, 1 \mathrm{~mm}$ dNTP mixture, $1 \mathrm{U} / \mu$ l of RNase inhibitor, $0.25 \mathrm{U} / \mu$ l of avian myeloblastosis virus-derived reverse transcriptase, and $0.125 \mu \mathrm{M}$ of oligo $\mathrm{d}(\mathrm{T})$-adaptor primer. Using a thermal cycler (Gene Amp PCR System, 2400-R; Perkin Elmer, Norwalk, Connecticut), the reaction mixture was incubated at $42^{\circ} \mathrm{C}$ for 30 minutes, heated at $99^{\circ} \mathrm{C}$ for 5 minutes, and then cooled to $5^{\circ} \mathrm{C}$ for 5 minutes.

\section{PCR}

One microliter of the RT products, containing $50 \mathrm{ng}$ of reverse-transcribed total RNA, was amplified by adding $20 \mu \mathrm{l}$ of PCR reaction mixture containing $10 \mathrm{~mm}$ Tris- $\mathrm{HCl}(\mathrm{pH} 8.3), 50 \mathrm{~mm} \mathrm{KCl}, 2.5 \mathrm{U} / 100 \mu \mathrm{l}$ of TaKaRa TaqDNA polymerase, with $0.2 \mu \mathrm{m}$ of a set of 20- to 22-mer oligonucleotide primers either for RhoA, RhoB, or RhoC and for G3PDH cDNA. Primers were synthesized to encompass a specific segment of the cDNA sequence of the RhoA (Suwa et al, 1998) (sense, 5'-CTGGTGATTGTTGGTGATGG-3'; antisense, 5'GCGATCATAATCTTCCT GCC-3'; spanning 183 bp), RhoB (sense, 5'-TGCTGATCGTGTTCAGTAAG-3'; antisense, 5'-AGCACATGAGAATGA CGTCG-3'; spanning $189 \mathrm{bp}$ ), RhoC (sense, 5'-TCCTCATCGTCTTCAGCAAG-3'; antisense, 5'-GAGGATGACATCAGTGTCCG-3'; spanning 181 bp), or G3PDH (sense, 5'ACGACCACTTTGTCAAGCTC-3'; antisense 5'-TCACA GTTGCCATGTAGACC-3'; spanning 226 bp between exons 7 and 8). The corresponding cDNA fragments were denatured at $94^{\circ} \mathrm{C}$ for 30 seconds, annealed at $58^{\circ} \mathrm{C}$ for 1 minute, and extended at $72^{\circ} \mathrm{C}$ for 1 minute. After 30 cycles of amplification, the PCR products were analyzed on a $2 \%$ agarose gel, and the bands were visualized using ethidium bromide during exposure to a UV transilluminator. The density of the bands on the gel was quantified by densitometric analysis using a Quantity One Scan System (ATTO, Tokyo, Japan). Gene expression was presented by the relative yield of PCR product from the target sequence 
to that from the G3PDH gene. Mean values from three independent experiments were taken as results.

\section{Real-Time PCR}

To confirm the results of RT-PCR, mRNA expression was also analyzed using real-time PCR (Roche LightCycler; Roche Diagnostics, Mannheim, Germany) in four benign, four borderline, and seven carcinoma cases. A master-mix of the following reaction components was prepared to the indicated endconcentration: $6.4 \mu \mathrm{l}$ of water, $1.2 \mu \mathrm{l}$ of $\mathrm{MgCl}_{2}$ (4 mM), $0.2 \mu$ l of forward primer $(0.4 \mathrm{M}), 0.2 \mu \mathrm{l}$ of reverse primer $(0.4 \mathrm{M})$ and $1.0 \mu \mathrm{l}$ of LightCycler Fast Start DNA Master SYBR Green I (Roche Diagnostics). Nine microliters of master-mix was added to the glass capillaries; $1 \mu \mathrm{l}$ volume, containing $50 \mathrm{ng}$ reverse-transcribed total RNA, was added as a PCR template. The capillaries were closed, centrifuged, and placed into the rotor. To improve SYBR Green I quantification, the temperature fluorescence measurement point was set at $72^{\circ} \mathrm{C}$. At the completion of cycling, melting curve analysis was performed to establish the specificity of the amplicons produced. The levels of expression of each mRNA and their estimated crossing points in each sample were determined relative to the standard preparation using the Light Cycler computer software. A ratio of specific mRNA/G3PDH amplification was then calculated, to correct for any differences in efficiency. The relative abundance of the mRNAs, expressed as fold changes, was extrapolated from crossing point data. A difference of 1 PCR cycle in crossing point number translates into a 2-fold change in mRNA expression.

\section{Western Blotting}

Tissues were lysed in a lysis buffer: $50 \mathrm{~mm}$ Tris- $\mathrm{HCl}$, $\mathrm{pH} 8.0,0.25 \mathrm{M} \mathrm{NaCl}, 0.5 \%$ NP-40, 1 mm phenylmethylsulfonyl fluoride (Sigma, St. Louis, Missouri), $1 \mu \mathrm{g} / \mathrm{ml}$ aprotinin (Boehringer Mannheim, Mannheim, Germany), $1 \mu \mathrm{g} / \mathrm{ml}$ leupeptin (Boehringer Mannheim), and 20 $\mu \mathrm{g} / \mathrm{ml}$ TPCK (Boehringer Mannheim). The lysates were centrifuged at $13,000 \times g$ for 20 minutes at $4^{\circ} \mathrm{C}$ and the supernatants were stored at $-80^{\circ} \mathrm{C}$. Extracts equivalent to $30 \mu \mathrm{g}$ of total protein were separated by SDS-PAGE ( $8 \%$ acrylamide) and transferred onto nitrocellulose membranes (Hybond TM-C Super, Amersham, Buckinghamshire, UK). The membranes were blocked in TBST (0.2 M NaCl, $10 \mathrm{~mm}$ Tris, $\mathrm{pH} 7.4,0.2 \%$ Tween-20) containing 5\% nonfat dry milk and $0.02 \%$ $\mathrm{NaN}_{3}$ for 1 hour and then incubated with mouse mAb against RhoA, Rho GDI, and ROCK (Santa Cruz Biotechnology, Santa Cruz, California) in TBST containing $5 \%$ nonfat dry milk. The membranes were then incubated with sheep anti-mouse Ig (Amersham) in TBST containing $2 \%$ nonfat dry milk. Bound antibody was detected with an enhanced chemiluminescence system (Amersham).

\section{Immunohistochemistry}

Immunohistochemical staining for RhoA was performed for 109 cases of ovarian tumors using the same mAb against RhoA (Santa Cruz) as used for Western blotting. Immunostaining was performed on paraffin sections by the avidin-biotin-peroxidase complex method, using a Histofine SAB-PO detector kit (Nichirei, Tokyo, Japan). In brief, the sections were deparaffinized in xylene and rehydrated through graded alcohols, then boiled in $0.01 \mathrm{M}$ citrate buffer (pH 6.0) for 15 minutes in a microwave oven. Next, $0.3 \%$ hydrogen peroxide was applied to block the endogenous peroxide activity, and the sections were incubated with normal goat serum to reduce nonspecific binding. They were then incubated with specific antibodies used at a 1:100 dilution or control nonimmunized mouse serum at $4^{\circ} \mathrm{C}$ overnight. Biotinylated goat anti-mouse IgG was used as a linker. After washing, the streptavidin-biotin complex was applied and stained with diaminobenzidine. Counterstaining was performed lightly with hematoxylin. Specific staining for RhoA was identified as a brown color in the cytoplasm. All of the control slides yielded negative results. The intensity of cytoplasmic staining was scored as strong $(+++)$, moderate $(++)$, faint $(+)$, or negative $(-)$, by comparison with the positive internal control; myoepithelial cells and vascular smooth muscle cells were moderately positive for RhoA (Kleer et al, 2002; van Golen et al, 1999). The evaluation of immunostaining was performed by two independent observers (T. I. and I. K.), who were unaware of the fate of the patient or the tissue site.

\section{Cell Culture}

The ovarian cancer cell line, SKOV3, was purchased from American Type Culture Collection (Rockville, Maryland). SKOV3 cells were cultured in DMEM (Gibco BRL, Grand Island, New York) with 5\% FCS (Biological Industries, Grand Island, New York) and $1 \%$ antibiotic-antimycotic solution (Gibco BRL). Incubation was performed at $37^{\circ} \mathrm{C}$ under $5 \% \mathrm{CO}_{2}$ in air. For RT-PCR and Western blotting, the cultured cells were transferred to a $75-\mathrm{cm}^{2}$ flask $\left(5 \times 10^{5}\right.$ cells/flask $)$ and incubated at $37^{\circ} \mathrm{C}$ under $20 \% \mathrm{O}_{2}$.

\section{ADP-Ribosylation Assay}

ADP-ribosylation was performed as described previously (Chardin et al, 1989; Fritz and Aktories, 1994) with $30 \mu \mathrm{g}$ of protein being incubated for 1 hour at $37^{\circ} \mathrm{C}$ with $20 \mathrm{ng}$ of $\mathrm{C} 3$ in $10 \mathrm{~mm}$ HEPES buffer, $\mathrm{pH} 8.0$, containing $15 \mathrm{~mm}$ isonicotinic acid hydrazide, $15 \mathrm{~mm}$ thymidine, $1 \mathrm{~mm}$ ATP, and $2 \mu \mathrm{M}\left[{ }^{32} \mathrm{P}\right] \mathrm{NAD}$. The reaction was stopped by the addition of $0.06 \mathrm{M}$ Tris- $\mathrm{Hcl}, \mathrm{pH}$ 7.0, 15\% (v/v) glycerol, 5\% $\beta$-mercaptoethanol, 2.3\% SDS, and $0.001 \%$ bromophenol blue. The mixture was then subjected to $12 \%$ SDS-PAGE. Then the gel was dried and radioactivity was determined using a MacBAS system (Fuji Film, Tokyo, Japan).

\section{In Vitro Invasion Assay}

Cell invasion through reconstituted basement membrane Matrigel was assayed by a method reported previously (Albini et al, 1987). Briefly, polycarbonate 
membranes (8.0- $\mu \mathrm{m}$ pore size) of the upper compartment of transwell culture chambers were coated with 5\% Matrigel (Becton Dickinson Labware, Bedford, Massachusetts). Subconfluent cells were starved for 24 hours and harvested with $0.05 \%$ trypsin containing $0.02 \%$ EDTA, washed twice with PBS, and resuspended at $10^{6}$ cells $/ \mathrm{ml}$ in serum-free medium with $0.1 \%$ BSA. The cell suspension $(100 \mu l)$ was placed in the upper compartment, and the lower compartment was immediately filled with $600 \mu \mathrm{l}$ of serum-free medium containing $0.1 \%$ BSA in the absence or presence of $10^{-7}$ or $10^{-6} \mathrm{M}$. After 20 hours of incubation, the cells on the upper surface of the filter were removed carefully with a cotton swab, the membranes were stained with Diff-Quik solution (Kokusai-Shiyaku, Kobe, Japan), and the cells that had migrated through the membrane to the lower surface were counted in six different fields under a light microscope at $\times 400$ magnification. Each experiment was performed in triplicate wells and repeated three times. Because LPA is known to stimulate the small GTPase Rho, the role of Rho in LPA-induced cancer cell invasion was tested by suppressing Rho function with C3 (a specific inhibitor of Rho). SKOV3 cells were pretreated with 0.1 $\mu \mathrm{g} / \mathrm{ml}$ C3 for 24 hours in the presence of $10 \%$ fetal bovine serum and resuspended at $10^{6} \mathrm{cells} / \mathrm{ml}$ in serum-free medium with $0.1 \%$ BSA. The cell suspension $(100 \mu \mathrm{l})$ was placed in the upper compartment.

\section{Statistical Analyses}

Statistical analysis was performed with the KruskalWallis rank test; Scheffe and Mann-Whitney's $U$ tests were used to compare expression levels in RT-PCR, real-time $\mathrm{PCR}$, immunohistochemistry, and invasion assay. The $p$ value obtained from the Mann-Whitney's $U$ test was described throughout the text. Wilcoxon signed-rank test was used to evaluate the differences in expression between primary and metastatic lesions. Spearman's rank correlation was used for the mRNA expression of RhoA, RhoB, and RhoC. A correlation coefficient greater 0.4 or less than -0.4 was considered significant. Differences were considered to be significant when $p<0.05$.

\section{References}

Albini A, Iwamoto Y, Kleinman HK, Martin GR, Aaronson JM, Kozlowski JM, and McEwan RN (1987). A rapid in vitro assay for quantitating the invasive potential of tumor cells. Cancer Res 47:3239-3245.

Avraham $\mathrm{H}$ and Weinberg RA (1989). Characterization and expression of the human rhoH12 gene product. Mol Cell Biol 9:2058-2066.

Baserga R (1994). Oncogenes and the strategy of growth factors. Cell 79:927-930.

Bernstein LR and Liotta LA (1994). Molecular mediators of interactions with extracellular matrix components in metastasis and angiogenesis. Curr Opin Oncol 6:106-113.

Chardin P, Boquet P, Madaule P, Popoff MR, Rubin EJ, and Gill DM (1989). The mammalian $G$ protein rhoC is ADPribosylated by Clostridium botulinum exoenzyme $\mathrm{C} 3$ and affects actin microfilaments in Vero cells. EMBO J 8:10871092.

Chomczynski P and Sacchi N (1987). Single-step method of RNA isolation by acid guanidinium thiocyanate-phenolchloroform extraction. Anal Biochem 162:156-159.

Clark EA, Golub TR, Lander ES, and Hynes RO (2000). Genomic analysis of metastasis reveals an essential role for RhoC. Nature 406:532-535.

Fishman DA, Liu Y, Ellerbroek SM, and Stack MS (2001). Lysophosphatidic acid promotes matrix metalloproteinase (MMP) activation and MMP-dependent invasion in ovarian cancer cells. Cancer Res 61:3194-3199.

Fritz G and Aktories K (1994). ADP-ribosylation of Rho proteins by Clostridium botulinum exoenzyme $\mathrm{C} 3$ is influenced by phosphorylation of Rho-associated factors. Biochem J 300:133-139.

Fritz G, Just I, and Kaina B (1999). Rho GTPases are over-expressed in human tumors. Int J Cancer 81:682-687.

Goetzl EJ and An S (1998). Diversity of cellular receptors and functions for the lysophospholipid growth factors lysophosphatidic acid and sphingosine 1-phosphate. FASEB J 12:1589-1598.

Hall A (1998). Rho GTPases and the actin cytoskeleton. Science 279:509-514.

Horiuchi A, Imai T, Shimizu M, Oka K, Wang C, Nikaido T, and Konish I (2002). Hypoxia-induced changes in the expression of VEGF, HIF- $1 \alpha$ and cell cycle-related molecules in ovarian cancer cells Anticancer Res 22:2697-2702.

Horiuchi A, Nikaido T, Ito K, Zhai Y, Orii A, Taniguchi S, and Fujii S (1998). Reduced expression of calponin h1 in leiomyosarcoma of the uterus. Lab Invest 78:839-846.

Hu YL, Tee MK, Goetzl EJ, Auersperg N, Mills GB, Ferrara N, and Jaffe RB (2001). Lysophosphatidic acid induction of vascular endothelial growth factor expression in human ovarian cancer cells. J Natl Cancer Inst 93:762-768.

Jaffe $A B$ and Hall A (2002). Rho GTPases in transformation and metastasis. In: Vande Woude $G$ and Klei G, editors. Advances in cancer research, vol 84. San Diego: Academic Press, 57-80.

Kamai T, Arai K, Tsujii T, Honda M, and Yoshida K (2001). Overexpression of RhoA mRNA is associated with advanced stage in testicular germ cell tumour. BJU Int 87:227-231.

Kleer CG, van Golen KL, Zhang Y, Wu ZF, Rubin MA, and Merajver SD (2002). Characterization of RhoC expression in benign and malignant breast disease: A potential new marker for small breast carcinomas with metastatic ability. Am J Pathol 160:579-584.

Mandai M, Konishi I, Komatsu T, Mori T, Arao S, Nomura H, Kanda Y, Hiai H, and Fukumoto M (1995). Mutation of the nm23 gene, loss of heterozygosity at the nm23 locus and K-ras mutation in ovarian carcinoma: Correlation with tumour progression and nm23 gene expression. Br J Cancer 72:691-695.

Morrow CP and Curtin JP (1998). Tumor of the ovary: Neoplasms derived from celomic epithelium. In: Synopsis of gynecologic oncology. Fifth editor. New York: Churchill Livingstone.

National Institutes of Health (1994). Ovarian cancer: Screening, treatment, and follow up. NIH Consensus Statement 12:1-30. 
Schmalfeldt B, Prechtel D, Harting K, Spathe K, Rutke S, Konik E, Fridman R, Berger U, Schmitt M, Kuhn W, and Lengyel $E$ (2001). Increased expression of matrix metalloproteinase (MMP)-2, MMP-9, and the urokinase-type plasminogen activator is associated with progression from benign to advanced ovarian cancer. Clin Cancer Res 7:2396-2404.

Scully RE, Young RH, and Clement PB (1998). Surface epithelial-stromal tumors and serous tumors. In: Tumor of the ovary, maldeveloped gonads, fallopian tube, and broad ligament. Atlas of Tumor Pathology, Third series, Fascicle 23. Washington, DC: Armed Forces Institute of Pathology, 5179.

Skubitz AP (2002). Adhesion molecules. Cancer Treat Res 107:305-329.

Suwa H, Ohshio G, Imamura T, Watanabe G, Arii S, Imamura M, Narumiya S, Hiai H, and Fukumoto M (1998). Overexpression of the rhoC gene correlates with progression of ductal adenocarcinoma of the pancreas. Br J Cancer 77:147-152.

Van Aelst L and D'Souza-Schorey C (1997). Rho GTPases and signaling networks. Genes Dev 11:2295-2322.

van Golen KL, Davies S, Wu ZF, Wang Y, Bucana CD, Root $\mathrm{H}$, Chandrasekharappa S, Strawderman M, Ethier SP, and Merajver SD (1999). A novel putative low-affinity insulin-like growth factor-binding protein, LIBC (lost in inflammatory breast cancer), and RhoC GTPase correlate with the inflammatory breast cancer phenotype. Clin Cancer Res 5:25112519.

van Golen KL, Wu ZF, Qiao XT, Bao LW, and Merajver SD (2000). RhoC GTPase, a novel transforming oncogene for human mammary epithelial cells that partially recapitulates the inflammatory breast cancer phenotype. Cancer Res 60: 5832-5838.
Xu Y, Fang XJ, Casey G, and Mills GB (1995a). Lysophospholipids activate ovarian and breast cancer cells. Biochem $J$ 309:933-940.

Xu Y, Gaudette DC, Boynton JD, Frankel A, Fang XJ, Sharma A, Hurteau J, Casey G, Goodbody A, Mellors A, et al (1995b). Characterization of an ovarian cancer activating factor in ascites from ovarian cancer patients. Clin Cancer Res 1:1223-1232.

Xu Y, Shen Z, Wiper DW, Wu M, Morton RE, Elson P, Kennedy AW, Belinson J, Markman M, and Casey G (1998). Lysophosphatidic acid as a potential biomarker for ovarian and other gynecologic cancers. JAMA 280:719-723.

Yamamoto S, Konishi I, Mandai M, Kuroda H, Komatsu T, Nanbu K, Sakahara H, and Mori T (1997). Expression of vascular endothelial growth factor (VEGF) in epithelial ovarian neoplasms: Correlation with clinicopathology and patient survival, and analysis of serum VEGF levels. $\mathrm{Br} \mathrm{J}$ Cancer 76:1221-1227.

Yoshioka K, Matsumura F, Akedo H, and Itoh K (1998). Small GTP-binding protein Rho stimulates the actomyosin system, leading to invasion of tumor cells. J Biol Chem 27:51465154.

Yoshioka K, Nakamori S, and Itoh K (1999). Overexpression of small GTP-binding protein RhoA promotes invasion of tumor cells. Cancer Res 59:2004-2010.

Westermann AM, Havik E, Postma FR, Beijnen JH, Dalesio O, Moolenaar WH, and Rodenhuis S (1998). Malignant effusions contain lysophosphatidic acid (LPA)-like activity. Ann Oncol 9:437-442. 